was that of Addai and Mari, despite the fact that his commentary includes the institutional words in the anaphora (pp. 15-16). Indeed, Bar Zō'bī's predecessors (beginning with Pseudo-Narsai and PseudoGregory of Arbela) did include the Institutio in their commentaries because they kept in mind the two other Eucharistic liturgies of the Church of the East, those of Nestorius and Theodore of Mopsuestia. This fact could have contributed towards the formation of the pattern of liturgical commentary which requires quotation of the institutional words. However, in general, the commentary by Bar Z $\bar{o}^{\prime}$ bì looks as if it follows the Addai and Mari rather than the two other Eastern anaphoras. Seleznyov's hypothesis does agree with my own feelings, but we have to take into account that a detailed study of the commentary is still a task for the future.

Seleznyov provides a useful glossary of liturgical terms (pp. 207210), but this is not enough for being able to follow the liturgical commentary if you do not already possess a mental outline of the East Syrian Eucharistic liturgy. For instance, to readers accustomed to the Byzantine rite, it would be rather difficult to notice that "the prayer that Christ gave to his Church" (line 201, p. 58/59 txt/tr.) is the Pater that is to be sung in full before the anaphora.

The critical edition of such an important work is a serious achievement in the field of historical liturgy. The scholarly community will certainly be grateful to Nikolai Seleznyov.

B. Lourié

Saint Petersburg State University of Aerospace Instrumentation

\title{
DAS RUSSISCH-DEUTSCHE SEMINAR ,SIMON LÜDWIGOWITSCH FRANK: DER DEUTSCHE KONTEXT DER RUSSISCHEN PHILOSOPHIE“
}

Am 10-11. Oktober 2013 hat das russisch-deutsche Seminar an der Staatlichen Universität St. Petersburg stattgefunden. Das Seminar war russischem Religionsphilosophen Simon Lüdwigowitsch Frank (1877-1950) gewidmet. Das Schicksal Franks war nicht nur mit Russland, sondern auch mit Deutschland verbunden. Er lernte zuerst an 
der Universität Moskau und dann an den deutschen Universitäten (Heidelberg, München). In 1912-1917 Jahren unterrichtete Frank auf dem Lehrstuhl für Philosophie an der Universität Petrograd (gegenwärtig St. Petersburg). Nachdem Frank zusammem mit 45 anderen russischen Wissenschaftlern auf dem sog. „Philosophischen-Dampfer" in 1922 Jahr des Sowjetischen Russland verwiesen war, hat er bis zum 1937 in Deutschland gelebt. Er ist zunächst in Frankreich und 1945 in England umzogen. In seinen Schriften entwickelte Frank die Ideen der "All-Einheit-Philosophie“ von Wladimir Sergejewitsch Solowjow. Moderne Forscher vergleichen den Ansatz Franks oft mit der Fundamentalontologie von Martin Heidegger. Simon L. Frank hat sich mit der Grundlagenforschungen für die Sozialphilosophie und die Philosophie der Religion beschäftigt.

Als Organisatoren des Seminares „Simon Lüdwigowitsch Frank: der deutsche Kontext der russischen Philosophie“" waren die Philosophische Fakultät der Staatlichen Universität St. Petersburg (Russland), die Hochschule für Philosophie München (Deutschland) und International Fond of Supporting of Social Researches, Humanities, and educational Programs "Intersozic“. Das Programm des Seminares nahm nahezu 30 Vorträgen auf, die von den Diskussionen begleitet wurden. Am Seminar haben bekannte Fachleute für die russischen Philosophie aus sechs Ländern (Russland, Ukraine, Deutschland, Schweiz, Polen, Tschechien) teilgenommen.

Deutscher Kenner des philosophischen Erbes von Frank, Prof. Peter Ehlen aus der Hochschule für Philosophie München hat in seiner Rede „Das denkende Erlebnis“: über die Ontologie der religiösen Erfahrung von S. L. Frank" der Analyse der Religionsphilosophie des russischen Denkers Aufmerksamkeit geschenkt. Sofern eigentümliche Besonderheit der Philosophie Franks die Einheit der Anthropologie und der Ontologie ist, scheint das Nachdenken von Frank einerseits den Überlegungen seines deutschen Zeitgenossen Heidegger nah zu sein, der im seinen Hauptwerk "Sein und Zeit" das Sein aus dem menschlichen Dasein zu verstehen versuchte. Andererseits kann man die Schrift von Frank „Das Unergründliche“ doch als Konterprojekt der Fundamentalontologie gewissermaßen betrachten, weil Frank im Unterschied zum Heidegger das Verstehen des Menschen nicht wahrhaben will, ohne die Beziehung des Menschen zum Dasein des Gottes zu verstehen. Nach Meinung von Prof. Ehlen ist eine Einheit und eine Differenz des menschlichen und göttlichen Daseins eigentlich die zentrale Frage des Denkens von Frank. Diese Analogia 
Entis lässt dem Mensch den Gott durch eine apophatischen Methode begreifen, die Frank von Nikolaus Cusanus übernimmt und als Motto seiner Philosophie verkündet: attingitur inattingibile inattingibiliter. Die Seinsanalogie ermöglicht das unmittelbare "lebendige Wissen" (das „Wissen-Leben“) über die Anwesenheit des Gottes im Selbstdasein des Menschen.

Der Vortrag von Dr. habil., Prof. Aleksej Malinov (Philosophische Fakultät der Staatlichen Universität St. Petersburg) „Russischer Ontologismus als Kritik des deutschen Transzendentalismus" wurde eine eigentümliche Entwicklung und Ergänzung des Vortrags von Prof. Ehlen. Prof. Malinov meint, daß Franks Aufgabe, nämlich die Vereinigung von Ontologie und Erkenntnistheorie, im breiteren Kontext der philosophischen Anthropologie gelöst werden kann. Das Problem der Konzeptsbestimmung des "lebendige Wissen" denn hat Prof. Gennadij Aljaev aus der Technischen Universität in Poltava (Ukraine) in seiner Meldung beleuchtet.

Einige Referenten haben die Frage der Philosophie der Religion im Werk von S. Frank berührt. Die Professorin Dr. Barbara Hallensleben aus der Theologischen Fakultät der Universität Freiburg (Schweiz) hat die Schrift von Frank „Mit uns ist Gott" analysiert. Die Dozentin der Staatlichen Universität St. Petersburg Nadeshda Tschupachina, die sich auf diese Franks Arbeit auch konzertriert hat, hält, daß die religiöse Erfahrung bei Frank äußerst echt ist, aber solche Echtheit mit der Evidenz nicht übereinstimmt. Dr. philos. Lenka Naldonieva, die Universität Ostrava (Tschechien) vertreten hat, hat einen Vortrag zum Thema „Die Ideen vom Übermensch und Gottesmensch bei Frank im Kontext des geschichtssophischen Prozess" gehalten. Frau Naldonieva ist zum Schluß gekommen, daß für Frank was den Menschen menschlich macht, $d$. h. der Grund der Menschlichkeit im Menschen, seine Gottesmenschlichkeit ist. Das höchste Gericht der menschlichen Existenz ist darum Gott und die Beziehung zu Gott ist ein Zeichen des menschlichen Wesens. D. theol. Yirshni Hoblik aus Universität Jana Evangelista Purkinein Usti nad Labem (Tschechien) hat zum Abschluß seiner Rede "Religiöse Wurzeln des Individualitätsbegriffs bei Frank" betont, daß die Philosophie Franks von groBem Wert ist, weil sie in dem Bemühen, die Entfremdung von Gott zu überwinden, die Entfremdung selbst gesehen werden kann.

In den Berichten und Besprechungen von Teilnehmer des Seminars war der Denknachlaß von Frank in der Perspektive der europäischen philosophischen Tradition dargestellt. Prof. Oleg Duschin aus 
der Staatlichen Universität St. Petersburg hat eine Besonderheit der Auslegung Franks des ontologischen Argument gezeigt. Die Schwester Teresa Obolevich aus der Päpstlichen Universität Johannes Paul II. In Krakau (Polen) hat in ihrer Mitteilung „Vom Neokantionismus zum Ontologismus" die Evolution der Ansichten von Frank verfolgt. In Vortrag „Religiöse Basis russischer Weltanschaung bei Frank und Masaryk" hat die Doktorin Ylija Kolesnychenko (Universität Jana Evangelista Purkinein Usti nad Labem, Tschechien) die Vergleichsanalyse der Theorien des russischen und tschechischen Denker unternommen. Herr Dennis Stammer, M. A. aus der Hochschule für Philosophie München (Deutschland) entdeckt eine Kongenialität der Doktrinen Whiteheads und Franks, die seiner Meinung nach in ihnen eigenen Ansatzen zum Subjekt eine "dynamische Ontologie“ aufgebaut haben. Prof. Igor Evlampiev (Philosophische Fakultät der Staatlichen Universität St. Petersburg) hat in seiner Nachricht „Frank und die mystische Tradition der europäischen Philosophie“ detailliert erklärt, warum die Religionsphilosophie von Frank dem mystischen Pantheismus zuzurechen ist. Die Doktorin Tatjana Resvich aus Orthodoxe Universität St. Tikhon (Moskau, Russland) hat die Zuhörer mit dem Problem der Zeit bekannt gemacht, wie Frank dieses Thema ausgearbeitet hat. Die Dozentin Lidija Dovydenko aus Kaliningrad hat dem Begriff der Transfinität der Realität bei Frank Aufmerksamkeit zugewandt.

In der Mitteilung von Professor Guido Vergauwen, Direktor des Instituts für Ökumenische Studien, Rektor der Universität Freiburg (der Schweiz) war die Analyse der christlichen gesellschaftlischen Lehre in modernen Säkulargesellschaft vorgestellt. Die Moral- und Sozialproblematik wurde auch zum Forschungsgegenstand von Dozentin Elena Krasnuchina (Philosophische Fakultät der Staatlichen Universität St. Petersburg), die ein Verständnis der Frage der Ich-DuBeziehung von Frank und Buber gegenüberstellt hat. Frau Ludmila Ornatskaja, Dr. philos. der Staatlichen Universität St. Petersburg hat im thematisch nahen Vortrag "Ich und Wir: Frank über die ontologische Grundlage der Gesellschaft" ihre Überlegung so zusammengefasst: „Das menschliche Leben in all seinen Bereichen (Familie, Wirtschaft, Wissenschaft, Religion etc.) ist bei Frank ein Ausdruck der ontologischen Einheit des menschlichen Seins. Der Mensch ist von Natur aus nur als Mitglied der Gesellschaft denkbar".

Im Rahmen des betreffenden Themas ist der Vortrag des Professors von Alexander Brodskij (Philosophische Fakultät der Staatlichen 
Universität St. Petersburg) unter dem provokativen Namen „Die Moral und die Gewalt in Ethik von Frank" überraschend ertönt, der eine ausgiebige Diskussion zur Folge gehabt hat. Das besondere Interesse bei Publikum hat Prof. Sergej Kibalnik aus dem Institut für russische Literatur in St. Petersburg (Pushkinskij Dom) erweckt, der in senem Referat Frank als Literaturforscher vorgestellt hat. Die Dozentin Elena Ovtschinnikova aus der Staatlichen Universität St. Petersburg hat im Vortrag „Die Formierung der Ethik als Studiendisziplin“ den Einfluss der deutschen philosophischen Tradition auf die Herausbildung der theoretischen Ethik in Russland in 18. und 19. Jahrhunderte demonstriert. Die Doktorin Oxana Nazarova (Hochschule für Philosophie München, Deutschland) hat eine summarische Übersicht über die modernen deutschen Forschungen des Werks von S. L. Frank gemacht.

Tagungsbericht von A. Malinov

Saint Petersburg State University 